Rosany Bochner 1

Claudio José Struchiner 2

\title{
Acidentes por animais peçonhentos e sistemas nacionais de informação
}

\author{
Recording of venomous bites and stings \\ by National Information Systems in Brazil
}

\footnotetext{
1 Centro de Informação Científica e Tecnológica, Fundação Oswal do Cruz. Av. Brasil 4365,

Rio de Janeiro, RJ 21045-900, Brasil. rosany@cict.fiocruz.br 2 Programa de Computação Científica, Fundação Oswaldo Cruz. Av. Brasil 4365 , Rio de Janeiro, RJ 21045-900, Brasil. stru@malaria.procc.fiocruz.br
}

\begin{abstract}
This paper highlights the epidemiol ogical surveillance of venomous bites and stings according to four national information systems: SINAN (National Databank of Major Causes of Morbi dity), SI NITOX (Nati onal Information System on Poisoning), SIH-SUS (Hospital Information System of the Unified Health System), and SIM (Mortality Information System). The authors conclude that each information system has specific characteristics and addresses different demands. Although they contain large amounts of data, even if combined they fail to reflect the real magnitude of disorders caused by venomous bites and stings in the country.

Key words Poisonous Animals; Information Systems; Epidemi ologic Surveillance; Epi demiology

Resumo Nestetrabalho foram analisados, sob a ótica da vigilância epidemiológica dos acidentes por ani mais peçonhentos, quatro sistemas naci onais de informação, o SINAN (Si stema de Informações de Agravos de Noti fi cação), o SI NITOX (Sistema Nacional de Informações Tóxi co-Farmacol ógi cas), o SIH-SUS (Sistema de Informações Hospitalares do Si stema Ú nico de Saúde) e o SIM (Sistema de Informações sobre Mortalidade). Concluiu-se que esses si stemas possuem características próprias, foram criados para atender demandas diferentes e apesar de produzi rem um grande vol ume de dados, não conseguem, ainda que analisados em conjunto, dar conta da di mensão real desses aci dentes.
\end{abstract}

Palavras-chave Animais Venenosos; Sistemas de Informação; Vigilância Epidemiológica; Epidemiologia 


\section{Introdução}

Apesar da longa tradição do Brasil no campo do Ofidismo, somente em junho de 1986, e em decorrência da crise na produção de soro no país, que culminou com a morte de uma criança em Brasília, foi implantado o Programa Nacional de Ofidismo na antiga Secretaria Nacional de Ações Básicas em Saúde do Ministério da Saúde (SNABS/MS), dando início a uma nova etapa no controle dos acidentes por animais peçonhentos. Nessa época, os acidentes ofídicos passam a ser de notificação obrigatória no país, e dados sobre escorpionismo e araneísmo começam a ser coletados a partir de 1988 (Cardoso, 1993).

É importante salientar, que a obrigatoriedade das notificações estava intimamente ligada à crise na produção de soro, pois uma das estratégias adotadas pelo MS para enfrentar o problema, foi a aquisição integral dos soros produzidos, que implicou na racionalização da oferta do produto, em nível nacional, e o estabelecimento de cotas de soros antiofídicos para as Secretarias Estaduais de Saúde, de acordo com a demanda estimada para cada estado (CNCZAP, 1991).

Atualmente, com a produção de soro estabilizada e atendendo de forma satisfatória à demanda, houve um afrouxamento na exigência da obrigatoriedade da notificação, estando a distribuição do soro não mais rigorosamente condicionada ao registro dos casos. Além disso, a adoção do SINAN (Sistema de Informações de Agravos de Notificação) em 1995, pela Coordenação Nacional de Controle de Zoonoses e Animais Peçonhentos (CNCZAP), gerou uma reação negativa por parte dos municípios e estados, os quais se mostraram resistentes à adoção do novo sistema, acabando por não enviar seus dados a essa coordenação, gerando uma visível quebra de continuidade, passando de 34.218 casos de acidentes por animais peçonhentos registrados em 1995, para 19.624 em 1996, 5.744 em 1997 e 7.119 em 1998 (Carvalho, 1997).

A carência de informações coletadas por essa coordenação através do SINAN, único sistema nacional que possui um módulo específico para tratar desse tipo de agravo à saúde, justifica a análise de outros sistemas nacionais de informação, que contemplam o registro de acidentes por animais peçonhentos, para verificar se são capazes de gerar informações necessárias e suficientes para se conhecer a dimensão real desse problema.

I remos considerar neste trabal ho, além do SINAN, o SINITOX (Sistema Nacional de Informações Tóxico-Farmacológicas), o SIH-SUS
(Sistema de Informações Hospitalares do Sistema Ú nico de Saúde) e o SIM (Sistema de Informações sobre Mortalidade), com o objetivo de analisar e comparar cada um desses sistemas sob a ótica da vigilância epidemiológica dos acidentes por animais peçonhentos, enfocando os seguintes aspectos: (a) finalidade e cobertura; (b) coleta e disponibilização de dados; (c) pontos fracos e fortes para a geração de informações úteis para se proceder a vigilância epidemiológica desse tipo de agravo à saúde e (d) casos e óbitos disponibilizados por esses sistemas para o período de 1979 a 1999.

\section{Metodologia}

De posse de publicações, CD-ROM s, Internet, artigos técnicos e fichas de coleta de dados, foram analisadas as características de cada um desses sistemas, a estrutura de suas fichas de coleta de dados e as variáveis por eles disponibilizadas.

Com base nessas análises, foi construída uma tabela comparativa (Tabela 1) que apresenta as características de cada um desses sistemas, como natureza dos dados que considera, órgão gestor, cobertura programada e realizada, instrumento de coleta de dados, número de variáveis coletadas e disponibilizadas, verificando aquelas de interesse para a análise epidemiológica dos acidentes por animais peçonhentos, tipo de disponibilização, anos disponíveis, meio de disponibilização, periodicidade, órgão responsável pela disponibilização e especificidade. Esse quadro aponta também os pontos fracos e fortes de cada um dos sistemas com relação à sua utilização para ações voltadas à vigilância epidemiológica desses acidentes.

Analisou-se também, a distribuição temporal de casos e óbitos em cada um dos sistemas (Tabelas 2 e 3), o que forneceu material para a discussão sobre a tendência desses acidentes ao longo do tempo, para a comparação entre os dados aportados por cada um dos sistemas e também para a construção de hipóteses acerca do que pode estar relacionado com o comportamento desses números.

Os casos de acidentes por animais peçonhentos, publicados nos Informes Epidemiológicos do SUS, são provenientes da CNCZAP. No período de 1986 a 1994, os dados referem-se às notificações encaminhadas pelas Secretarias Estaduais de Saúde à CNCZAP. De 1995 em diante, com a adoção do SI NAN pela CNCZAP, os dados referem-se às informações encaminhadas à essa coordenação através do sistema SINAN, somadas com as que chegam por ou- 
Comparação dos sistemas nacionais de informação sobre acidentes por animais peçonhentos.

\begin{tabular}{|c|c|c|c|c|}
\hline & SINAN & SINITOX & SIH-SUS & SIM \\
\hline Natureza & $\begin{array}{l}\text { Casos de agravos } \\
\text { de notificação. } \\
\text { Contempla doenças de } \\
\text { diferentes naturezas, } \\
\text { crônicas transmissíveis } \\
\text { (AIDS, hanseníase, } \\
\text { tuberculose, } \\
\text { esquistossomose) e não } \\
\text { transmissíveis } \\
\text { (pneumoniose, desnutrição } \\
\text { grave), além de agudas } \\
\text { transmissíveis } \\
\text { (imunopreviníveis, cólera, } \\
\text { dengue) e não } \\
\text { transmissíveis (acidentes } \\
\text { com animais peçonhentos, } \\
\text { intoxicação por agrotóxicos). }\end{array}$ & $\begin{array}{l}\text { Casos de intoxicação } \\
\text { e envenenamento. } \\
\text { Até 1998, considerou } 13 \\
\text { categorias de agentes } \\
\text { tóxicos (medicamentos, } \\
\text { animais peçonhentos, } \\
\text { animais não peçonhentos, } \\
\text { produtos químicos } \\
\text { industriais, pesticidas } \\
\text { agropecuários, pesticidas } \\
\text { domésticos, raticidas, } \\
\text { domissanitários, produtos } \\
\text { de toalete, plantas, } \\
\text { alimentos, outros produtos } \\
\text { e não determinado). } \\
\text { A partir de 1999, foram } \\
\text { incluídos produtos } \\
\text { veterinários, metais, drogas } \\
\text { de abuso e outros animais } \\
\text { venenosos, além de separar } \\
\text { os animais peçonhentos. }\end{array}$ & $\begin{array}{l}\text { Casos de internações } \\
\text { hospitalares. } \\
\text { Foi concebido para operar } \\
\text { o sistema de pagamento } \\
\text { de internação dos hospitais } \\
\text { contratados pelo Ministério } \\
\text { da Previdência. Em 1986, } \\
\text { foi estendido aos hospitais } \\
\text { filantrópicos, em 1987, aos } \\
\text { universitários e de ensino, } \\
\text { e em 1991, aos hospitais } \\
\text { públicos municipais, } \\
\text { estaduais e federais, nesse } \\
\text { último caso, somente aos } \\
\text { da administração indireta } \\
\text { e de outros ministérios. }\end{array}$ & $\begin{array}{l}\text { Óbitos fetais e não fetais. } \\
\text { As Secretarias de Saúde } \\
\text { coletam as DO dos cartórios } \\
\text { e dão entrada, no SIM, das } \\
\text { informações nelas contidas. } \\
\text { A causa básica de óbito, } \\
\text { constitui uma das informações } \\
\text { primordiais, que é codificada } \\
\text { a partir do declarado pelo } \\
\text { médico atestante, segundo } \\
\text { regras estabelecidas pela } \\
\text { Organização Mundial de } \\
\text { Saúde. }\end{array}$ \\
\hline Órgão gestor & CENEPI & FIOCRUZ & SAS & CENEPI \\
\hline $\begin{array}{l}\text { Cobertura } \\
\text { programada }\end{array}$ & $\begin{array}{l}\text { Universal. } \\
\text { Casos registrados pelas } \\
\text { Coordenações Estaduais } \\
\text { do Programa Nacional de } \\
\text { Controle de Acidentes por } \\
\text { Animais Peçonhentos. }\end{array}$ & $\begin{array}{l}\text { Casos notificados por uma } \\
\text { rede de } 32 \text { Centros de } \\
\text { Controle de Intoxicações, } \\
\text { localizados em } 17 \text { estados. }\end{array}$ & $\begin{array}{l}\text { Casos de internações } \\
\text { registrados pelos hospitais } \\
\text { públicos e conveniados } \\
\text { ao SUS. }\end{array}$ & $\begin{array}{l}\text { Universal. } \\
\text { Óbitos registrados pelas } \\
\text { Secretarias Estaduais } \\
\text { de Saúde. }\end{array}$ \\
\hline Cobertura realizada & $\begin{array}{l}\text { De } 1986 \text { a } 1993 \text { e em 1995, } \\
\text { contou com a participação } \\
\text { de todos os Estados. Em } \\
\text { 1994, não contabilizou os } \\
\text { dados dos Estados de } \\
\text { Roraima e da Paraíba. Em } \\
\text { 1996, não contabilizou os } \\
\text { dados dos Estados do AM, } \\
\text { PB, BA, MS e GO. Em 1997, } \\
\text { não contabilizou os dados } \\
\text { dos Estados de RO, AC, RR, } \\
\text { PA, AP, MA, PI, PB, SE, ES, } \\
\text { SP, MS e GO. Em 1998, não } \\
\text { contabilizou os dados dos } \\
\text { Estados de RO, AC, RR, PA, } \\
\text { AP, MA, PI, SE, MG, MT. }\end{array}$ & $\begin{array}{l}\text { Limitada pelo número } \\
\text { insuficiente de Centros, } \\
\text { a notificação não é } \\
\text { compulsória e o envio dos } \\
\text { dados pelos Centros ao } \\
\text { SINITOX ocorre de forma } \\
\text { espontânea, como pode } \\
\text { ser observado pelos números } \\
\text { de Centros em atividade } \\
\text { no país e participantes da } \\
\text { estatística do SINITOX, } \\
\text { respectivamente, para os } \\
\text { anos de } 1985 \text { a } 1999 ; \\
1985 \text { - } 14 \text { e } 14 ; \\
1986 \text { - } 18 \text { e } 13 ; \\
1987 \text { - } 19 \text { e } 12 ; \\
1988 \text { - } 20 \text { e } 15 ; \\
1989 \text { - } 24 \text { e } 17 ; \\
1990 \text { - } 25 \text { e } 19 ; \\
1991 \text { - } 27 \text { e } 21 ; \\
1992 \text { - } 29 \text { e } 23 ; \\
1993 \text { - } 29 \text { e } 23 ; \\
1994 \text { - } 29 \text { e } 25 ; \\
1995 \text { - } 31 \text { e } 26 ; \\
1996 \text { - } 30 \text { e } 29 ; \\
1997 \text { - } 30 \text { e } 30 ; \\
1998 \text { - } 32 \text { e } 32 ; \\
1999 \text { - } 32 \text { e } 29\end{array}$ & $\begin{array}{l}\text { Registra cerca de } 70 \% \\
\text { a } 80 \% \text { das internações } \\
\text { ocorridas no país. } \\
\text { Atualmente, com a } \\
\text { expansão dos seguros } \\
\text { privados e planos de } \\
\text { medicina de grupo, } \\
\text { pode-se dizer que essa } \\
\text { variação é de fato desigual } \\
\text { para os diversos tipos de } \\
\text { procedimentos, estando } \\
\text { provavelmente a cobertura } \\
\text { de partos e outros atos de } \\
\text { menor custo, em torno de } \\
80 \% \text { e sendo maior que } \\
90 \% \text { nos casos de } \\
\text { procedimentos de mais } \\
\text { alta complexidade/custo, } \\
\text { não cobertos por esses } \\
\text { planos (Carvalho, 1997). } \\
\text { Reúne dados sobre todas } \\
\text { as internações remuneradas } \\
\text { pelo SUS, significando, } \\
\text { aproximadamente, 15 } \\
\text { milhões de internações/ } \\
\text { ano (Travassos, 1997). }\end{array}$ & $\begin{array}{l}\text { Registra cerca de } 80 \% \text { dos } \\
\text { óbitos, devido à perda de DO } \\
\text { nos órgãos responsáveis e, } \\
\text { principalmente, pelo } \\
\text { sepultamento em cemitérios } \\
\text { clandestinos (Carvalho, 1997). }\end{array}$ \\
\hline
\end{tabular}

(continua) 
Tabela 1 (continuação)

\begin{tabular}{|c|c|c|c|c|}
\hline & SINAN & SINITOX & SIH-SUS & SIM \\
\hline $\begin{array}{l}\text { Instrumento } \\
\text { de coleta }\end{array}$ & $\begin{array}{l}\text { Ficha específica para } \\
\text { Notificação de Acidente } \\
\text { por Animais Peçonhentos } \\
\text { (FUNASA, 1998). }\end{array}$ & $\begin{array}{l}\text { Ficha de Notificação e de } \\
\text { Atendimento. Centros de } \\
\text { Atendimento Toxicológico } \\
\text { (SINITOX, 2001). }\end{array}$ & $\begin{array}{l}\text { Ficha de AIH (INAMPS, } \\
\text { 1986). }\end{array}$ & DO (UNIFESP, 2001). \\
\hline $\begin{array}{l}\text { Número de variáveis } \\
\text { coletadas através } \\
\text { desses instrumentos }\end{array}$ & 49 & 59 & 73 & 62 \\
\hline $\begin{array}{l}\text { Número e } \% \text { de } \\
\text { variáveis coletadas } \\
\text { de interesse }\end{array}$ & $\begin{array}{l}39 \\
(80 \%)\end{array}$ & $\begin{array}{l}44 \\
(75 \%)\end{array}$ & $\begin{array}{l}18 \\
(25 \%)\end{array}$ & $\begin{array}{l}36 \\
(58 \%)\end{array}$ \\
\hline $\begin{array}{l}\text { Número de variáveis } \\
\text { disponibilizadas }\end{array}$ & 3 (UF, Tipo de Animal, Data). & $\begin{array}{l}10 \text { (Centro, Região, Mês } \\
\text { ou Trimestre, Vítima, Agente } \\
\text { Tóxico, Causa ou } \\
\text { Circunstância, Faixa Etária, } \\
\text { Sexo, Zona, Evolução). }\end{array}$ & $\begin{array}{l}\text { Número variável ao longo } \\
\text { dos anos, sendo para os } \\
\text { arquivos anuais reduzidos, } \\
\text { distribuídas da seguinte } \\
\text { forma (Ano - no): } \\
1993-35 ; 1994-39 \\
1995-40,1996-41 \\
1997-42,1998-41 \\
\text { e } 1999-52 \text {. }\end{array}$ & $\begin{array}{l}50 \text {, sendo que algumas dessas } \\
\text { variáveis passaram a ser } \\
\text { coletadas apenas a partir de } \\
\text { determinada época, variando } \\
\text { de acordo com a informação } \\
\text { e com a UF, estando, } \\
\text { portanto, em branco antes } \\
\text { deste período. }\end{array}$ \\
\hline $\begin{array}{l}\text { Número e \% } \\
\text { de variáveis } \\
\text { disponibilizadas } \\
\text { de interesse }\end{array}$ & $\begin{array}{l}3 \\
(100 \%)\end{array}$ & $\begin{array}{l}10 \\
(100 \%)\end{array}$ & $\begin{array}{l}23 \text { ( } 5 \text { construídas } \\
\text { e não coletadas) } \\
\text { (44\%) com base em } 52 \\
\text { variáveis coletadas }\end{array}$ & $\begin{array}{l}26 \\
(52 \%)\end{array}$ \\
\hline $\begin{array}{l}\text { Tipo de } \\
\text { disponibilização }\end{array}$ & $\begin{array}{l}\text { Tabelas com os casos } \\
\text { distribuídos por UF, tipo do } \\
\text { agravo, mês e ano. }\end{array}$ & $\begin{array}{l}\text { Tabelas com os casos } \\
\text { distribuídos por agente } \\
\text { tóxico, segundo centro, } \\
\text { região, vítima, mês (1991 a } \\
\text { 1994) ou trimestre (a partir } \\
\text { de 1995), causa (1985 a } \\
\text { 1998) ou circunstância (a } \\
\text { partir de 1999), faixa etária } \\
\text { (com } 5 \text { categorias em 1985, } \\
7 \text { de } 1986 \text { a 1991 e } 13 \text { a } \\
\text { partir de 1992), sexo, zona } \\
\text { (a partir de 1993), evolução } \\
\text { (com } 3 \text { categorias de 1986 } \\
\text { a 1998 e } 7 \text { a partir de 1999). } \\
\text { Tabelas com os óbitos } \\
\text { distribuídos, a partir de } \\
\text { 1993, por agente tóxico } \\
\text { segundo região, causa ou } \\
\text { circunstância, sexo, faixa } \\
\text { etária e centro (a partir de } \\
\text { 1996). }\end{array}$ & $\begin{array}{l}\text { Arquivos contendo } \\
\text { internações, realizadas em } \\
\text { hospitais públicos e } \\
\text { conveniados ao SUS. }\end{array}$ & $\begin{array}{l}\text { Arquivos contendo óbitos } \\
\text { fetais e não fetais. }\end{array}$ \\
\hline Anos disponíveis & 1986 a 1998. & 1985 a 1999. & 1984 a 1999. & 1979 а 1998. \\
\hline Periodicidade & Trimestral. & Anual. & Anual e Mensal. & Anual. \\
\hline $\begin{array}{l}\text { Meio de } \\
\text { disponibilização }\end{array}$ & $\begin{array}{l}\text { Informe Epidemiológico } \\
\text { do SUS. }\end{array}$ & $\begin{array}{l}\text { Estatística Anual de Casos } \\
\text { de Intoxicação e } \\
\text { Envenenamento (1985 } \\
\text { a 1999). } \\
\text { Internet (1997 a 1999). }\end{array}$ & $\begin{array}{l}\text { CD-ROM das AlH: anuais } \\
\text { (arquivos reduzidos, com } \\
\text { as informações principais, } \\
\text { de } 1993 \text { a 1999) e mensais } \\
\text { (arquivos completos, de } \\
\text { novembro de } 1994 \text { a agosto } \\
\text { de 2000). Internet (dados } \\
\text { anuais de 1984-1991 e } \\
\text { mensais de janeiro de } 1992 \\
\text { a julho de 2001). }\end{array}$ & $\begin{array}{l}\text { CD-ROM (1979-1998). } \\
\text { Internet (1979-1998). }\end{array}$ \\
\hline
\end{tabular}

(continua) 


\begin{tabular}{|c|c|c|c|c|}
\hline & SINAN & SINITOX & SIH-SUS & SIM \\
\hline $\begin{array}{l}\text { Responsável pela } \\
\text { disponibilização }\end{array}$ & CENEPI & FIOCRUZ & DATASUS & DATASUS \\
\hline Especificidade & $\begin{array}{l}\text { Dispõe de um módulo } \\
\text { específico para esse tipo } \\
\text { de agravo à saúde. }\end{array}$ & $\begin{array}{l}\text { Não é específico para esse } \\
\text { tipo de agravo à saúde. }\end{array}$ & $\begin{array}{l}\text { Não é específico para esse } \\
\text { tipo de agravo à saúde. }\end{array}$ & $\begin{array}{l}\text { Não é específico para esse } \\
\text { tipo de agravo à saúde. }\end{array}$ \\
\hline $\begin{array}{l}\text { Pontos fracos à sua } \\
\text { utilização para ações } \\
\text { voltadas à vigilância } \\
\text { epidemiológica } \\
\text { de acidentes por } \\
\text { animais peçonhentos }\end{array}$ & $\begin{array}{l}\text { Baixa cobertura. } \\
\text { Inconsistência de dados } \\
\text { anuais com mensais. } \\
\text { Dados sempre sujeitos } \\
\text { à revisão. } \\
\text { Número insuficiente de } \\
\text { variáveis disponibilizadas } \\
\text { para se proceder a uma } \\
\text { análise mais completa } \\
\text { do agravo. } \\
\text { Defasagem na } \\
\text { disponibilização dos dados. } \\
\text { Não contempla, em sua } \\
\text { ficha de coleta de dados, } \\
\text { a espécie do animal agressor } \\
\text { e o endereço do local } \\
\text { do acidente. } \\
\text { Não disponibiliza os óbitos } \\
\text { registrados. } \\
\text { O sistema é pouco flexível, } \\
\text { não permitindo a inclusão } \\
\text { de variáveis ou elaboração } \\
\text { de relatórios personalizados } \\
\text { (Carvalho, 1997). }\end{array}$ & $\begin{array}{l}\text { Não possui informações de } \\
\text { nove Estados (AC, AM, RR, } \\
\text { RO, TO, AL, MA, PI, SE) e } \\
\text { do DF. Apresenta tendência } \\
\text { do número de casos, } \\
\text { influenciada pela } \\
\text { participação dos Centros } \\
\text { em suas estatísticas. } \\
\text { Seus dados não constituem } \\
\text { uma amostra representativa } \\
\text { da incidência deste tipo de } \\
\text { agravo à saúde, pois a } \\
\text { maioria dos Centros está } \\
\text { localizada em capitais, o } \\
\text { que pode ser um fator de } \\
\text { subnotificação para alguns } \\
\text { desses acidentes, que são } \\
\text { mais freqüentes no interior } \\
\text { ou em zonas rurais do } \\
\text { estado. Até } 1998, \text { os } \\
\text { animais não eram separados } \\
\text { em serpentes, aranhas e } \\
\text { escorpiões. A partir de } \\
\text { 1995, os dados passam a } \\
\text { ser disponibilizados por } \\
\text { trimestre e não mais por } \\
\text { mês, o que prejudica a } \\
\text { realização de análises } \\
\text { temporais. Não contempla } \\
\text { em sua ficha de coleta, } \\
\text { variáveis importantes para } \\
\text { a análise desse tipo de } \\
\text { acidente, tais como local } \\
\text { do corpo atingido, } \\
\text { manifestações clínicas, } \\
\text { número de ampolas de soro } \\
\text { utilizado, exames realizados. }\end{array}$ & $\begin{array}{l}\text { Registra apenas os casos } \\
\text { que sofreram internação, ou } \\
\text { seja, os casos mais graves. } \\
\text { Questionamento sobre a } \\
\text { qualidade dos dados que } \\
\text { aporta, em decorrência de } \\
\text { fraudes e manipulações } \\
\text { pelos prestadores de } \\
\text { serviços. Não admite } \\
\text { correções posteriores à } \\
\text { realização do pagamento, } \\
\text { mesmo que tenham sido } \\
\text { verificados erros de } \\
\text { digitação ou codificação. } \\
\text { O sistema não identifica } \\
\text { reinternações e } \\
\text { transferências de outros } \\
\text { hospitais, levando a } \\
\text { eventuais duplas ou triplas } \\
\text { contagens de um mesmo } \\
\text { paciente (Carvalho, 1997). } \\
\text { Não registra o município } \\
\text { de ocorrência do acidente } \\
\text { e demais variáveis } \\
\text { importantes para a análise } \\
\text { desse tipo de agravo à } \\
\text { saúde, tais como gênero e } \\
\text { espécie do animal, local do } \\
\text { corpo atingido, tipo de } \\
\text { soro utilizado, número de } \\
\text { ampolas, data e hora } \\
\text { do acidente. } \\
\text { Inconsistência entre os } \\
\text { diagnósticos principal e } \\
\text { secundário, a partir da } \\
\text { adoção da CID-10. }\end{array}$ & $\begin{array}{l}\text { Registra apenas os óbitos. } \\
\text { Retardo em seu } \\
\text { processamento, sendo } \\
\text { atualmente, a defasagem } \\
\text { entre a ocorrência do óbito } \\
\text { e sua disponibilização, de } \\
\text { mais de dois anos. } \\
\text { Acentuado número de óbitos } \\
\text { por sintomas e sinais mal } \\
\text { definidos. Preenchimento } \\
\text { inadequado (em branco ou } \\
\text { ignorado) de diversos campos } \\
\text { da DO, sendo a mudança } \\
\text { freqüente do seu modelo um } \\
\text { dos principais responsáveis } \\
\text { (Carvalho, 1997; Oliveira \& } \\
\text { Pereira, 1997). }\end{array}$ \\
\hline
\end{tabular}

(continua)

tras vias, como aerogramas, relatórios mensais (Carvalho, 1997). Apesar da base de dados do SINAN para acidentes por animais peçonhentos só ter sido iniciada em 1995, foi considerado neste trabalho a série histórica de 1986 a 1998, como fazendo parte desse sistema.

Dessa forma, os casos registrados pela CNCZAP no período de 1986 a 1998, aqui associados ao SINAN, foram obtidos através de dois fascículos da publicação Informe Epidemiológico do SUS (CENEPI, 1998, 1997).

Os casos do SINITOX se referem aos anos de 1985 a 1999, e os óbitos aos anos de 1986 a 1999, ambos obtidos a partir de suas publicações anuais e respectivas revisões (SI NITOX, 1992, 1993, 1995, 1997, 1998a, 1998b, 1999a, 1999b,
2000; PRONITOX, 1990, 1991; SNITF, 1986, 1987, 1988, 1989). É importante salientar que até 1998, inclusive, o SI NITOX não desagregava os dados sobre acidentes por animais peçonhentos em serpentes, aranhas e escorpiões.

Os sistemas SIH-SUS e SIM , utilizam o Código Internacional de Doenças - 9a Revisão (CID-9 - OMS, 1985) e a 10a Revisão (CID-10 OMS, 1993) para fornecer os diagnósticos principal e secundário das internações (SIH-SUS) e a causa básica do óbito (SIM). O SIH-SUS utilizou a CID-9 até o ano de 1997, passando para a CID-10 a partir de 1998. Já o SIM adotou a CID10 a partir de 1996.

Segundo a CID-9, os códigos relacionados aos acidentes por animais peçonhentos, tanto 
Tabela 1 (continuação)

\begin{tabular}{|c|c|c|c|c|}
\hline & SINAN & SINITOX & SIH-SUS & SIM \\
\hline $\begin{array}{l}\text { Pontos fortes à sua } \\
\text { utilização para ações } \\
\text { voltadas à vigilância } \\
\text { epidemiológica } \\
\text { de acidentes por } \\
\text { animais peçonhentos }\end{array}$ & $\begin{array}{l}\text { Constitui o único sistema } \\
\text { de informação que possui } \\
\text { um módulo específico para } \\
\text { este tipo de agravo à saúde. } \\
\text { Apresenta coordenações } \\
\text { estaduais em todos os } \\
\text { Estados, o que facilita a } \\
\text { captação de casos de todo } \\
\text { o país. }\end{array}$ & $\begin{array}{l}\text { A disponibilização de seus } \\
\text { dados é realizada de forma } \\
\text { regular, registrando os casos } \\
\text { desse tipo de acidente, } \\
\text { independente de ter sido } \\
\text { ou não internado. } \\
\text { Prevê em sua ficha de } \\
\text { coleta, o registro da } \\
\text { espécie do animal e o } \\
\text { endereço do local do } \\
\text { acidente, informações } \\
\text { importantes para a } \\
\text { realização do mapeamento } \\
\text { desses animais. }\end{array}$ & $\begin{array}{l}\text { A disponibilização de seus } \\
\text { dados é realizada de forma } \\
\text { rápida e regular. } \\
\text { Apresenta a maior parte das } \\
\text { informações coletadas. } \\
\text { Com a adoção da CID-10, } \\
\text { a partir de 1998, é possível } \\
\text { obter o local do acidente } \\
\text { através do 4o dígito } \\
\text { utilizado para as categorias } \\
\text { do Capítulo XX, referente } \\
\text { a Causas Externas de } \\
\text { Morbidade e Mortalidade, } \\
\text { onde } 0 \text { - residência; } \\
1 \text { - habitação coletiva; } \\
2 \text { - escolas, outras } \\
\text { instituições e áreas de } \\
\text { administração pública; } \\
3 \text { - área para a prática de } \\
\text { esportes e atletismo; } 4 \text { - rua } \\
\text { e estrada, } 5 \text { - área de } \\
\text { comércio e serviços; } \\
6 \text { - áreas industriais em } \\
\text { construção; } 7 \text { - fazenda; } \\
8 \text { - outros locais } \\
\text { especificados; } 9 \text { - local não } \\
\text { especificado (OMS, 1993). } \\
\text { Fornece arquivos e não } \\
\text { tabelas fechadas. }\end{array}$ & $\begin{array}{l}\text { Constitui o sistema que possui } \\
\text { a maior cobertura dos óbitos } \\
\text { ocorridos no país. Apresenta } \\
\text { o registro de variáveis } \\
\text { relacionadas a óbitos por } \\
\text { causas externas, como local } \\
\text { do acidente, se foi ou não } \\
\text { acidente de trabalho, etc. } \\
\text { Com a adoção da CID-10, a } \\
\text { partir de } 1996 \text {, é possível } \\
\text { obter o local do acidente } \\
\text { através do } 4 \text { o dígito utilizado } \\
\text { para as categorias do Capítulo } \\
\text { XX, referente a Causas } \\
\text { Externas de Morbidade e } \\
\text { Mortalidade, onde } \\
0 \text { - residência; } 1 \text { - habitação } \\
\text { coletiva; } 2 \text { - escolas, outras } \\
\text { instituições e áreas de } \\
\text { administração pública; } \\
3 \text { - área para a prática de } \\
\text { esportes e atletismo; } 4 \text { - rua } \\
\text { e estrada, } 5 \text { - área de } \\
\text { comércio e serviços; } \\
6 \text { - áreas industriais em } \\
\text { construção; } 7 \text { - fazenda; } \\
8 \text { - outros locais } \\
\text { especificados; } 9 \text { - local não } \\
\text { especificado (OMS, 1993). } \\
\text { Fornece arquivos e não } \\
\text { tabelas fechadas. }\end{array}$ \\
\hline
\end{tabular}

SINAN = Sistema de Informações de Agravos de Notificação; SINITOX = Sistema Nacional de Informações

Tóxico-Farmacológicas; SIH-SUS = Sistema de Informações Hospitalares - Sistema Único de Saúde; SIM = Sistema de Informações sobre Mortalidade; CENEPI = Centro Nacional de Epidemiologia; DO = Declaração de Óbito;

FIOCRUZ = Fundação Oswaldo Cruz; SAS = Secretaria de Assistência à Saúde; AIH = Autorização de Internação

Hospitalar; DATASUS = Departamento de Informática do SUS; UF = Unidades da Federação; CID-10 = Décima

Revisão da Classificação Internacional das Doenças; $A C=A c r e ; A L=$ Alagoas; $A M=A m a z o n a s ; A P=A m a p a ́ ;$

$\mathrm{BA}=$ Bahia; $\mathrm{DF}=$ Distrito Federal; $\mathrm{ES}=$ Espírito Santo; GO = Goiás; MA = Maranhão; MG = Minas Gerais;

$\mathrm{MS}=$ Mato Grosso do Sul; MT = Mato Grosso; PA = Pará; PB = Paraíba; PI = Piauí; RO = Rondônia; RR = Roraima;

$\mathrm{SE}=$ Sergipe; $\mathrm{SP}=$ São Paulo; $\mathrm{TO}=$ Tocantins

para o registro de morbidade como de mortalidade, são encontrados na Classificação Suplementar de Causas Externas de Lesões e Envenenamentos, e devem ser considerados com quatro dígitos a fim de se obter a discriminação do animal envolvido: E905.0 - intoxicação e reações tóxicas causadas por serpentes e lagartos venenosos, E905.1 - intoxicação e reações tóxicas causadas por aranhas venenosas e E905.2 - intoxicação e reações tóxicas causadas por escorpião (OMS, 1985).

Com respeito à CID-10, os códigos relacionados aos acidentes por animais peçonhentos, para o registro de morbidade, são encontrados no Capítulo XIX: Lesões e Envenenamentos e al gumas outras conseqüências de Causas Externas e no Capítulo XX: Causas Externas de Morbidade e Mortalidade. Os códigos referentes ao Capítulo XIX, são também considerados com quatro dígitos para se obter a discriminação do animal envolvido: T63.0 - veneno de serpente, T63.2 - veneno de escorpião eT63.3 veneno de aranha e os códigos referentes ao Capítulo XX, são considerados com apenas três dígitos, pois neste caso o quarto dígito não é indicador do animal envolvido, mas sim do local onde ocorreu o acidente: X20 - contato com serpentes e lagartos venenosos, X21 - contato com aranhas venenosas e X22 - contato com escorpiões. Para o registro de mortalidade, são utilizados apenas os códigos relacionados aos acidentes por animais peçonhentos pertencentes ao Capítulo XX, ou seja, X20, X21 e X22 (OMS, 1993).

O SIH-SUS conta com três tipos de formulários de Autorização de Internação Hospitalar $(\mathrm{AlH})$, tipos 1 , 3 e 5 . O tipo 1, é de preenchimento obrigatório em qualquer internação e contém um número que identifica aquela internação, que é copiado nos formulários tipo 3 e 5 em caso de ser necessário seu preenchimento. Dessa forma, o total de registros no 
banco de dados corresponde ao total de formulários de AlH, dos tipos 1, 3 e 5, preenchidos pelos hospitais, enquanto o total de formulários do tipo 1, corresponde ao total de internações (Travassos, 1996).

Para a obtenção dos casos e óbitos referentes ao SIH-SUS, utilizamos os CD-ROMs anuais referentes ao Movimento de Autorização de Internação Hospitalar - Arquivos Reduzidos, disponíveis para os anos de 1993 a 1999 (DATASUS, 1993, 1994, 1995, 1996, 1997, 1998, 1999). Para os anos de 1993 a 1996, foram criados arquivos para serpentes, aranhas e escorpiões, contendo todas as variáveis disponibilizadas pelo sistema. Os arquivos foram construídos a partir da seleção dos registros, que apresentavam como identificação da AlH o tipo 1 e diagnóstico principal compatível com o acidente e animal agressor envolvido, sendo o diagnóstico secundário não utilizado, uma vez que não estava disponível para esses anos, em se tratando de arquivos anuais. Para os anos de 1997 a 1999, foram também construídos arquivos para serpentes, aranhas e escorpiões, contendo todas as variáveis disponibilizadas pelo sistema, sendo que dessa vez, a seleção dos casos de interesse foi realizada com base na identificação da AlH e nos diagnósticos principal e secundário, ou seja, para a construção do arquivo de serpentes para o ano de 1998, foram selecionados apenas os registros que apresentavam a identificação da AlH do tipo 1 e os códigos X20 ou T63.0, para o diagnóstico principal ou o secundário. Com exceção do ano de 1997, que ainda utilizava a CID-9, foram constatados problemas no preenchimento desses campos, gerando inconsistências importantes. Por exemplo, no arquivo de serpentes, foram contabilizados casos causados por aranhas, escorpiões, outros répteis, outros artrópodes, peixes, outros animais marinhos, outros animais venenosos e animal venenoso não especificado.

Para minimizar esses problemas, pois o mesmo caso era contabilizado em dois arquivos diferentes, foi necessário criar um critério para separar os casos referentes à cada animal. Esse critério, poderia ser baseado dando prioridade ao que é declarado no diagnóstico principal ou ao código T63 em detrimento ao código $X$.

Para efeito desse trabalho, foi definido o seguinte critério: dar prioridade ao diagnóstico principal, contabilizando serpentes, aranhas e escorpiões em seus próprios arquivos, ou seja, retirando-os dos outros, e o que for referente aos demais animais citados, mantê-los em seus arquivos de origem. Por exemplo, no arquivo de serpentes, baseado no diagnóstico principal, retirar apenas os registros que apresentam em seu diagnóstico principal, os códigos referentes a aranhas e escorpiões, ou seja, X21, X22, T63.2 eT63.3.

Para a obtenção dos óbitos disponibilizados pelo SIM , utilizamos o CD-ROM referente ao Sistema de Informação sobre Mortalidade (DATASUS, 2001) e construímos arquivos para serpentes, aranhas e escorpiões para os anos de 1979 a 1998, contendo todas as variáveis disponibilizadas pelo sistema, sendo a causa básica do óbito utilizada para selecionar os óbitos de interesse.

Com base nos arquivos de internações e óbitos construídos para serpentes, aranhas e escorpiões, foi possível obter o número de internações causadas por cada um desses animais, e os óbitos relacionados a essas internações para os anos de 1993 a 1999, o número de óbitos decorrentes de acidentes por cada um desses animais para os anos de 1979 a 1998, bem como analisar as variáveis disponibilizadas e a qualidade de seu preenchimento.

\section{Resultados}

A comparação dos quatro sistemas de informação e a apresentação para cada um deles dos pontos fracos e fortes, relacionados à sua utilização para ações voltadas à vigilância epidemiológica de acidentes por animais peçonhentos, encontram-se na Tabela 1.

Segundo a cobertura programada, existem dois sistemas universais (SINAN e SI M) e dois sistemas limitados, o SI NITOX, com Centros de Controle de Intoxicações localizados em apenas 17 estados brasileiros e o SIH-SUS, que cobre somente as internações hospitalares ocorridas no país com financiamento do SUS. Por outro Iado, a cobertura realizada fica muito aquém da programada para o SINAN e também para o SINITOX, já o SIH-SUS apresenta praticamente a mesma cobertura programada, ou seja, 70 a $80 \%$ das internações realizadas no país, e o SIM tem uma sub-notificação de quase $20 \%$, devido à perda de Declarações de Óbitos pelos órgãos responsáveis e também pelo sepultamento em cemitérios clandestinos (Tabela 1 ).

Foram contabilizados os números de variáveis, através dos instrumentos de coleta de dados de cada um dos sistemas, e dentre essas verificou-se o número e o percentual de variáveis de interesse para a análise dos acidentes por animais peçonhentos. Podemos notar que o SIH-SUS, é o sistema que coleta o mai or número de variáveis, porém, é o que apresenta o menor percentual de variáveis de interesse (25\%), 
já o SINAN apresenta o maior percentual de variáveis de interesse (80\%). O SINITOX coleta mais variáveis que o SINAN, o que já era de se esperar em se tratando de um sistema mais amplo, que registra casos de intoxicação e envenenamento referentes a diversos agentes tóxicos, porém, seu percentual de variáveis de interesse é menor (75\%). O SI M coleta o menor número de variáveis, contudo apresenta um percentual de variáveis de interesse maior que o do SIH-SUS (58\%), o que pode ser explicado pelo detalhamento que oferece para os óbitos relacionados a causas externas (Tabela 1 ).

Com base nos meios de disponibilização de dados desses sistemas, foram contabilizados os números de variáveis disponibilizadas por cada um deles, bem como o número e o percentual dessas variáveis que são de interesse para a análise desse tipo de acidente. Tanto o SINAN como o SINITOX, disponibilizam um número muito reduzido de variáveis, sendo todas de interesse. O número de variáveis disponibilizadas pelo SIH-SUS e pelo SIM é muito próximo, sendo o percentual de variáveis disponibiliza- das de interesse um pouco maior para o SIM (Tabela 1).

Observa-se, uma nítida quebra de continuidade dos dados associados ao SINAN a partir de 1996 (Tabela 2), e ao contrário desse comportamento de queda, o SINITOX vem apresentando uma série histórica ascendente, chegando a superar o número de casos registrados pelo SINAN a partir de 1997, o que é um indicador da fragilidade das informações apontadas pelo SINAN, dada a cobertura do SINITOX. Já o SIH-SUS, apresenta uma série histórica descendente, com uma queda mais acentuada em 1998, ano este em que é adotada pelo sistema a CID-10 em substituição à CID-9. No entanto, ao analisar os dados do SIH-SUS para cada um dos animais, podemos verificar que para aranhas e escorpiões ocorre exatamente o inverso, com aumento significativo no número de casos a partir de 1998. Em 1999, o SI NITOX passa a separar os animais peçonhentos em serpentes, aranhas e escorpiões, e o resultado é um comportamento que difere do que vem sendo observado no país desde 1988, apresentan-

Tabela 2

Casos registrados de acidentes por animais peçonhentos no Brasil, segundo os sistemas de informação SINAN,

SINITOX e SIH-SUS, distribuídos por ano de ocorrência.

\begin{tabular}{|c|c|c|c|c|c|c|c|c|c|c|c|}
\hline \multirow[t]{2}{*}{ Anos } & \multicolumn{4}{|c|}{ SINAN } & \multirow{2}{*}{\multicolumn{3}{|c|}{$\begin{array}{c}\text { SINITOX } \\
\text { Animais Peçonhentos }\end{array}$}} & \multicolumn{4}{|c|}{ SIH-SUS } \\
\hline & Serpente & Aranha & Escorpião & Total & & & & Serpente & Aranha & Escorpião & Total \\
\hline 1985 &.. &.. &.. &.. & & 4.657 & &.. &.. &.. & .. \\
\hline 1986 & 8.574 &.. &.. & 8.574 & & 7.079 & &.. &.. &.. & .. \\
\hline 1987 & 21.463 &.. &.. & 21.463 & & 6.665 & &.. &.. & .. & .. \\
\hline 1988 & 19.815 & 1.968 & 3.741 & 25.524 & & 6.248 & &.. &.. &.. & .. \\
\hline 1989 & 20.957 & 2.668 & 3.803 & 27.428 & & 6.875 & & .. &.. & .. & .. \\
\hline 1990 & 19.763 & 2.979 & 4.466 & 27.208 & & 8.359 & &.. &.. &.. & .. \\
\hline 1991 & 19.541 & 4.200 & 5.233 & 28.974 & & 10.382 & &.. &.. & .. & .. \\
\hline 1992 & 21.007 & 4.480 & 7.046 & 32.533 & & 12.881 & &.. &.. &.. & .. \\
\hline 1993 & 21.300 & 6.136 & 8.081 & 35.517 & & 12.643 & & 18.912 & 249 & 888 & 20.049 \\
\hline 1994 & 21.026 & 5.561 & 7.959 & 34.546 & & 13.744 & & 18.889 & 192 & 707 & 19.788 \\
\hline 1995 & 19.804 & 6.803 & 7.611 & 34.218 & & 12.889 & & 15.351 & 123 & 509 & 15.983 \\
\hline 1996 & 12.120 & 3.666 & 3.838 & 19.624 & & 14.177 & & 12.770 & 115 & 364 & 13.251 \\
\hline 1997 & 4.918 & 484 & 342 & 5.744 & & 18.051 & & 11.908 & 130 & 343 & 12.381 \\
\hline 1998 & 6.156 & 624 & 339 & 7.119 & & 19.433 & & 8.193 & 381 & 879 & 9.453 \\
\hline \multirow[t]{3}{*}{1999} & $\ldots$ & $\ldots$ & $\ldots$ & $\ldots$ & & $12.373^{*}$ & & 8.271 & 397 & 1.120 & 9.788 \\
\hline & & & & & Serpente & Aranha & Escorpião & & & & \\
\hline & & & & & $4.418^{\star}$ & $2.266^{\star}$ & $5.689^{*}$ & & & & \\
\hline
\end{tabular}

SINAN = Sistema de Informações de Agravos de Notificação; SINITOX = Sistema Nacional de Informações

Tóxico-Farmacológicas; SIH-SUS = Sistema de Informações Hospitalares - Sistema Único de Saúde.

Fonte: SINAN, Centro Nacional de Epidemiologia; SINITOX, Fundação Oswaldo Cruz; SIH-SUS, Departamento de Informática do SUS

* Dados sujeitos à revisão.

Não se aplica dado numérico.

.. Dado numérico não disponível. 
do número de acidentes por escorpiões maior que o por serpentes. Isso pode significar, uma melhor captação de casos relacionados a aranhas e escorpiões pelo SI NITOX, o que já era esperado, pela localização dos Centros ser preferencialmente em capitais e o fato desses acidentes serem mais comuns em áreas urbanas.

Podemos verificar, a partir de 1986, decréscimo no número de óbitos registrados pelo SIM para acidentes por animais peçonhentos (Tabela 3). É interessante salientar, que em junho desse mesmo ano, era implantado o Programa Nacional de Ofidismo, centralizando assim o controle desses acidentes no âmbito do MS. O número de óbitos registrados pelo SIHSUS também vem diminuindo, em contra partida, os óbitos do SINITOX oscilam ao longo do tempo, ora diminuindo, ora aumentando. É in- teressante verificar, que esse comportamento oscilante ocorre também com o número de óbitos registrados pelo SIM, tanto para aranhas como para escorpiões, o que pode ser mais um indicador de que o SI NITOX registra de forma mais abrangente os acidentes por aranhas e escorpiões. Chama a atenção, o número elevado de óbitos registrados pelo SIH-SUS em 1999, para acidentes por escorpiões, que não acompanha apenas o crescimento dos casos, uma vez que a letalidade quase que duplicou.

\section{Discussão}

Os casos disponibilizados pela CNCZAP, que neste trabalho estamos associando ao SINAN, apresentam dados estabilizados no período de

\section{Tabela 3}

Óbitos registrados de acidentes por animais peçonhentos no Brasil, segundo os sistemas de informação SINITOX, SIM e SIH-SUS, distribuídos por ano de ocorrência.

\begin{tabular}{|c|c|c|c|c|c|c|c|c|c|c|c|}
\hline \multirow[t]{2}{*}{ Anos } & \multirow{2}{*}{\multicolumn{3}{|c|}{$\begin{array}{c}\text { SINITOX } \\
\text { Animais Peçonhentos }\end{array}$}} & \multicolumn{4}{|c|}{ SIM } & \multicolumn{4}{|c|}{ SIH-SUS } \\
\hline & & & & Serpente & Aranha & Escorpião & Total & Serpente & Aranha & Escorpião & Total \\
\hline 1979 & \multicolumn{3}{|c|}{.. } & 261 & 4 & 24 & 289 &.. &.. &.. &.. \\
\hline 1980 & \multicolumn{3}{|c|}{.. } & 269 & 4 & 37 & 310 &.. &.. &.. &.. \\
\hline 1981 & \multicolumn{3}{|c|}{.. } & 241 & 4 & 27 & 272 &.. &.. &.. &.. \\
\hline 1982 & \multicolumn{3}{|c|}{.. } & 237 & 4 & 36 & 277 &.. &.. &.. &.. \\
\hline 1983 & \multicolumn{3}{|c|}{..} & 255 & 5 & 37 & 297 &.. &.. &.. &.. \\
\hline 1984 & \multicolumn{3}{|c|}{.. } & 251 & - & 23 & 274 &.. &.. &.. & .. \\
\hline 1985 & \multicolumn{3}{|c|}{..} & 259 & 4 & 29 & 292 & .. &.. &.. &.. \\
\hline 1986 & \multicolumn{3}{|c|}{68} & 268 & 4 & 20 & 292 &.. &.. &.. &.. \\
\hline 1987 & \multicolumn{3}{|c|}{41} & 207 & 2 & 42 & 251 &.. &.. &.. &.. \\
\hline 1988 & \multicolumn{3}{|c|}{27} & 188 & 1 & 47 & 236 &.. &.. &.. &.. \\
\hline 1989 & \multicolumn{3}{|c|}{25} & 159 & 6 & 40 & 205 &.. &.. &.. &.. \\
\hline 1990 & \multicolumn{3}{|c|}{25} & 151 & 7 & 43 & 201 &.. &.. &.. &.. \\
\hline 1991 & \multicolumn{3}{|c|}{42} & 124 & 7 & 47 & 178 &.. &.. &.. &.. \\
\hline 1992 & \multicolumn{3}{|c|}{48} & 125 & 5 & 39 & 169 &.. &.. &.. & .. \\
\hline 1993 & \multicolumn{3}{|c|}{38} & 128 & 5 & 27 & 160 & 81 & - & 11 & 92 \\
\hline 1994 & \multicolumn{3}{|c|}{43} & 119 & 5 & 39 & 163 & 76 & 1 & 3 & 80 \\
\hline 1995 & \multicolumn{3}{|c|}{32} & 145 & 4 & 31 & 180 & 72 & - & 3 & 75 \\
\hline 1996 & \multicolumn{3}{|c|}{55} & 107 & 7 & 20 & 134 & 51 & - & 8 & 59 \\
\hline 1997 & \multicolumn{3}{|c|}{57} & 96 & 8 & 35 & 139 & 46 & 3 & 6 & 54 \\
\hline 1998 & \multicolumn{3}{|c|}{73} & 95 & 9 & 22 & 126 & 45 & - & 8 & 53 \\
\hline 1999 & \multicolumn{3}{|c|}{$26^{*}$} & $\ldots$ & $\ldots$ & $\ldots$ & $\ldots$ & 37 & 4 & 19 & 60 \\
\hline & Serpente & Aranha & Esco & & & & & & & & \\
\hline & $15^{\star}$ & $2^{*}$ & & & & & & & & & \\
\hline
\end{tabular}

SINAN = Sistema de Informações de Agravos de Notificação; SINITOX = Sistema Nacional de Informações Tóxico-Farmacológicas;

SIH-SUS = Sistema de Informações Hospitalares - Sistema Único de Saúde; SIM = Sistema de Informações sobre Mortalidade;

Fonte: SINITOX, Fundação Oswaldo Cruz; SIM e SIH-SUS, Departamento de Informática do SUS.

* Dados sujeitos à revisão.

. Não se aplica dado numérico

... Dado numérico não disponível.

- Dado numérico igual a zero, não resultante de arredondamento. 
1990 a 1995, apontando uma média anual de 20.000 acidentes por serpentes, 5.000 por aranhas e 7.000 por escorpiões. A verificação desse comportamento estável por parte da CNCZAP, pode ter levado a um afrouxamento na exigência do envio de notificações por parte dos Estados à essa coordenação, levando a quebra de continuidade dos dados, observada a partir de 1996. Essa hipótese de estabilidade, pode também explicar o fato da não disponibilização a partir de 1999, dos acidentes por animais peçonhentos, nem no Informe Epidemiológico do SUS, que mudou seu perfil e deixou de divulgar dados, e nem no Bol etim El etrônico Epidemiológico, onde dados de doenças de notificação compulsória são apresentados. Outra explicação para essa quebra de continuidade, pode estar relacionada à adoção do SINAN pela CNCZAP a partir de 1995, que por sua vez teve muito pouca receptividade por parte dos municípios e estados, gerando dados fragilizados devido à baixa cobertura do sistema.

O comportamento dos casos registrados pelo SIH-SUS, vai contra a hipótese de estabilidade da incidência desses acidentes, pois vem apresentando diminuição no número de casos para os acidentes ofídicos, sendo mais acentuada a partir de 1998, e significativo aumento de acidentes por aranhas e escorpiões, também a partir deste mesmo ano. Essas mudanças, coincidem com a adoção pelo SIH-SUS da CID-10 em substituição a CID-9, o que gera alterações no registro dos diagnósticos principal e secundário. Também podemos pensar na diminuição das internações por serpentes, como fruto da melhoria da qualidade do atendimento no que se refere a uma melhor triagem dos casos, identificando acidentes por serpentes não peçonhentas, que não necessitam de sorologia e por sua vez de internação, baseado para isso nas manifestações clínicas do paciente, nos aspectos da lesão e também, se necessário, na aplicação de testes para detectar a presença de veneno.

Por outro lado, as internações por aranhas e escorpiões, que também vinham diminuindo, passam a aumentar significativamente em 1998, o que pode ser explicado pelo aumento da população desses animais ou pelo aumento da gravidade desses acidentes, ou mesmo por problemas de registro com a adoção da CID10, já citado anteriormente.

Podemos verificar através da Tabela 3, que a adoção da CID-10 pelo SIM, a partir de 1996, não gerou diferenças no comportamento dos dados, o que pode ser explicado pela compatibilidade entre os códigos da CID-9 com os da CID-10, utilizados para o registro de mortalidade $(E 905.0=X 20, E 905.1=X 21$ e E905.2 =X22).

\section{Conclusão}

Para se proceder a vigilância epidemiológica dos acidentes por animais peçonhentos, é imprescindível a existência de um sistema nacional de informação, integrado com os demais sistemas, com cobertura universal, com ficha de coleta de dados padronizada e específica para esse tipo de acidente, com um programa informatizado para a entrada dos dados da ficha, capaz de gerar relatórios e análises dos dados digitados em todos os níveis de atuação, com grande articulação com o nível central e disponibilização de todas as variáveis de interesse de forma regular e rápida.

Atualmente, apesar de dispormos de quatro sistemas nacionais que contemplam o registro de acidentes por animais peçonhentos, o que temos na verdade, são informações dissociadas umas das outras, fazendo com que a análise seja realizada sob diversos ângulos, sem conseguir no entanto dar conta da dimensão real desse problema. 


\section{Referências}

CARDOSO, J. L. C., 1993. Acidentes por Animais Peçonhentos na Coordenação de Zoonoses e Animais Peçonhentos - Comentários e Sugestões. Brasília: Ministério da Saúde. (mimeo.)

CARVALHO, D. M., 1997. Grandes sistemas nacionais de informação em saúde: Revisão e discussão da situação atual. Informe Epidemiológi co do SUS, 4:7-46.

CENEPI (Centro Nacional de Epidemiologia), 1997. Série histórica de casos de agravos e doenças infecciosas e parasitárias - Brasil - 1980 a 1996. Informe Epidemiológico do SUS, 1:33-38.

CENEPI (Centro Nacional de Epidemiologia), 1998. Casos de agravos e doenças infecciosas e parasitárias notificados de janeiro a dezembro de 1997 e igual período de 1998, por unidade federada, Brasil. Informe Epidemiológico do SUS, 4:55-60.

CNCZAP (Coordenação Nacional de Controle de Zoonoses e Animais Peçonhentos), 1991. Manual de Diagnósti co e Tratamento de Acidentes Ofídicos. Brasília: CNCZAP, Centro Nacional de Epidemiologia, Fundação Nacional de Saúde, Ministério da Saúde.

DATASUS (Departamento de Informática do SUS), 1993. Movimento de Autorização de Internação Hospitalar. Arquivos Reduzidos, 1993. CD-ROM. Brasília: Ministério da Saúde.

DATASUS (Departamento de Informática do SUS), 1994. Movimento de Autorização de Internação Hospitalar. Arquivos Reduzidos, 1994. CD-ROM. Brasília: Ministério da Saúde.

DATASUS (Departamento de Informática do SUS), 1995. Movimento de Autorização de Internação Hospitalar. Arquivos Reduzidos, 1995. CD-ROM. Brasília: Ministério da Saúde.

DATASUS (Departamento de Informática do SUS), 1996. Movimento de Autorização de Internação Hospitalar. Arquivos Reduzidos, 1996. CD-ROM. Brasília: Ministério da Saúde.

DATASUS (Departamento de Informática do SUS), 1997. Movimento de Autorização de Internação Hospitalar. Arqui vos Reduzidos, 1997. CD-ROM. Brasília: Ministério da Saúde.

DATASUS (Departamento de Informática do SUS), 1998. Movimento de Autorização de Internação Hospitalar. Arqui vos Reduzi dos, 1998. CD-ROM. Brasília: Ministério da Saúde.

DATASUS (Departamento de Informática do SUS), 1999. Movimento de Autorização de Internação Hospitalar. Arqui vos Reduzidos, 1999. CD-ROM. Brasília: Ministério da Saúde.

DATASUS (Departamento de Informática do SUS), 2001. Sistema de Informação sobre Mortalidade, 1979 - 1998. Dados de Declaração de Óbito. CDROM. Brasília: Ministério da Saúde.

FUNASA (Fundação Nacional de Saúde), 1998. Manual de Diagnóstico e Tratamento de Aci dentes por Animais Peçonhentos. Brasília: FUNASA, Ministério da Saúde

INAMPS (Instituto Nacional de Assistência Médica da Previdência Social), 1986. Autorização de Internação Hospitalar. Brasília: INAMPS, Ministério da Previdência e Assistência Social. (mimeo.)

OLIVEIRA, H. \& PEREIRA, I. P. A., 1997. Estatísticas de mortalidade e nascidos vivos: Considerações sobre principais problemas. Informe Epidemiológico do SUS, 3:15-19.

OMS (Organização Mundial de Saúde), 1985. Manual da Classificação Estatística Internacional de Doenças, Lesões e Causas de Óbito - Nona Conferência de Revisão. São Paulo: Centro da OMS para Classificação de Doenças em Português.

OMS (Organização Mundial da Saúde), 1993. Classificação Estatística Internacional de Doenças e Problemas Relacionados à Saúde, 10ạ Revisão. v. 1. São Paulo: Centro Colaborador da OMS para a Classificação de Doenças em Português.

PRONITOX (Programa Nacional Integrado de Informações Tóxico-Farmacológicas), 1990. Casos de Intoxi cação Atendidos pel os Centros de Toxi cologia. Brasil, 1989. Porto Alegre: PRONITOX, Fundação Oswaldo Cruz.

PRONITOX (Programa Nacional Integrado de Informações Tóxico-Farmacológicas), 1991. Estatística Anual deIntoxicação Humana. Centros de Informações Toxicológicas. Brasil, 1990. Rio de Janeiro: PRONITOX, Superintendência de Informação Científica eTecnológica, Fundação Oswaldo Cruz.

SNITF (Sistema Nacional de Informações TóxicoFarmacológicas), 1986. Intoxicações no Brasil. Morbidade e Mortalidade. Registro de Dados dos Centros de Informação Toxi cológica, 1985. Porto Alegre: SNITF, Fundação Oswaldo Cruz.

SNITF (Sistema Nacional de Informações TóxicoFarmacológicas), 1987. Relatório de Cadastro de Casos, 1986. Porto Alegre: SNITF, Fundação Oswaldo Cruz.

SNITF (Sistema Nacional de Informações TóxicoFarmacológicas), 1988. Coordenação do Sistema Nacional de Informações Tóxico-Farmacológicas. Estatística, 1987. Porto Alegre: SNITF, Fundação Oswaldo Cruz.

SNITF (Sistema Nacional de Informações TóxicoFarmacológicas), 1989. Estatística, 1988. Porto Alegre: SNITF, Fundação Oswaldo Cruz.

SINITOX (Sistema Nacional de Informações TóxicoFarmacológicas), 1992. Estatística Anual deCasos de Intoxicação e Envenenamento. Brasil, 1991. Rio de Janeiro: SINITOX, Centro de Informação Científica e Tecnológica, Fundação Oswaldo Cruz.

SINITOX (Sistema Nacional de Informações TóxicoFarmacológicas), 1993. Estatística Anual de Casos deIntoxicação e Envenenamento. Brasil, 1992. Rio de Janeiro: SINITOX, Centro de Informação Científica e Tecnológica, Fundação Oswaldo Cruz.

SI NITOX (Sistema Nacional de Informações TóxicoFarmacológicas), 1995. Estatística Anual de Casos deIntoxicação e Envenenamento. Brasil, 1993. Rio de Janeiro: SINITOX, Centro de Informação Científica e Tecnológica, Fundação Oswaldo Cruz.

SINITOX (Sistema Nacional de Informações TóxicoFarmacológicas), 1997. Estatística Anual deCasos deIntoxicação e Envenenamento. Brasil, 1995. Rio de Janeiro: SINITOX, Centro de Informação Científica e Tecnológica, Fundação Oswaldo Cruz.

SI NITOX (Sistema Nacional de Informações TóxicoFarmacológicas), 1998a. Revisão da Estatística Anual de Casos de Intoxicação e Envenenamento. 
Brasil, 1996. Rio de Janeiro: SINITOX, Centro de Informação Científica e Tecnológica, Fundação Oswaldo Cruz.

SINITOX (Sistema Nacional de Informações TóxicoFarmacológicas), 1998b. Revisão da Estatística Anual de Casos de Intoxicação e Envenenamento. Brasil, 1995. Rio de Janeiro: SI NITOX, Centro de Informação Científica e Tecnológica, Fundação Oswaldo Cruz.

SINITOX (Sistema Nacional de Informações TóxicoFarmacológicas), 1999a. Estatística Anual de Casos de Intoxicação e Envenenamento. Brasil, 1998. Rio de Janeiro: SINITOX, Centro de Informação Científica e Tecnológica, Fundação Oswaldo Cruz.

SINITOX (Sistema Nacional de Informações TóxicoFarmacológicas), 1999b. Revisão da Estatística Anual de Casos de Intoxicação e Envenenamento. Brasil, 1997. Rio de Janeiro: SINITOX, Centro de Informação Científica e Tecnológica, Fundação Oswaldo Cruz.
SI NITOX (Sistema Nacional de Informações TóxicoFarmacológicas), 2000. Estatística Anual de Casos deIntoxicação e Envenenamento. Brasil, 1999. Rio de Janeiro: SINITOX, Centro de Informação Científica e Tecnológica, Fundação Oswaldo Cruz.

SINITOX (Sistema Nacional de Informações TóxicoFarmacológicas), 2001. Manual de Preenchimento da Ficha de Notificação e de Atendimento. Centros de Informação, de Controlee de Atendimento Toxicológico. Rio de Janeiro: SINITOX, Centro de Informação Científica e Tecnológica, Fundação Oswaldo Cruz.

TRAVASSOS, C., 1996. O Sistema de Informações Hospitalares do Sistema Único de Saúde-SIH-SUS, Rio de Janeiro: Fundação Oswaldo Cruz. (mimeo.)

UNIFESP (Universidade Federal de São Paulo), 2001. Declaração deÓbito: \ttp:// gamba.epm.br/pub/ atestado/modelo.htm>.

Recebido em 25 de junho de 2001

Versão final reapresentada em 17 de outubro de 2001 Aprovado em 14 de dezembro de 2001 